\title{
The structure of charged d-dimensional stars: comparing different equations of state
}

\author{
J. D. Arbañil Vela* ${ }^{* \dagger}$ and V. T. Zanchin ${ }^{\dagger}$ \\ Centro de Ciências Naturais e Humanas, Universidade Federal do ABC, \\ Rua Santa Adélia 166, 09210-170 Santo André, SP, Brazil. \\ E-mail: jose.vela@ufabc.edu.br, zanchin@ufabc.edu.br
}

\begin{abstract}
In this work we review some of the general properties of static charged fluid in the context of the Einstein-Maxwell theory with cosmological term in four and higher dimensions. The metric is assumed to have a general form such that the geometry of the spatial section of the spacetime can be spherical, planar or hyperbolic. As first study we obtain the Tolman-Oppenheimer-Volkoff (TOV) equation, which describe the hydrostatic equilibrium, for a charged fluid in d-dimensional spacetime with cosmological constant for different geometries. In order to solve the resulting TOV equations, we need to provide an equation of state and an additional constraint for the charge density. For simplicity, the charge density is assumed to be proportional to the energy density, and we test such a set up for different charge fractions. We present and analyze numerical results showing the dependence of the star structure on the particular equation of state for two particular choices of equations of polytropic type.
\end{abstract}

5th International School on Field Theory and Gravitation,

April 20 - 242009

Cuiabá city, Brazil

\footnotetext{
${ }^{*}$ Poster Session

${ }^{\dagger}$ We thank Conselho Nacional de Desenvolvimento Científico e Tecnológico - CNPq, Brazil, and Coordenação de Aperfeiçoamento de Pessoal de Nível Superior - CAPES, Brazil, for scholarships.
} 


\section{Introduction}

Static solutions of Einstein-Maxwell equations have been studied by many authors. In particular, static solutions of charged dust fluids where the gravitational force is balanced by the repulsive electric forces are easy to find (see, e.g., refs. [1]-[3]). Such a kind of systems have physical properties that make them important in the context of theories for fundamental interactions. Hence it is interesting to investigate also the generalizations for higher dimensions, like was done by $[4,5]$. In this work we study some of the effects of the electric charge and extra dimensions in the structure of a static star. We begin by writing the equilibrium equations as a generalized version of the Tolman-Oppenheimer-Volkoff (TOV) equations for a charged fluid, in a $d$-dimensional spacetime with cosmological constant. The system of equations is close by choosing the charge density to be proportional to the energy density, and it is considered two different polytropic equations of state (EOS). Throughout this work we use $c=1$ and metric signature $2-d$.

\section{D-dimensional TOV equation with charge and cosmological constant}

The Einstein-Maxwell equations in $d$-dimensional spacetime with cosmological constant $\Lambda$ are

$$
\begin{gathered}
R_{\mu v}-\frac{1}{2} g_{\mu v} R+\Lambda g_{\mu v}=\frac{d-2}{d-3} S_{d-2} G_{d} T_{\mu v}, \\
\nabla_{v} F^{\mu v}=S_{d-2} J^{\mu}
\end{gathered}
$$

with $R_{\mu v}$ and $R$ being the Ricci tensor and scalar, respectively. The constant $S_{d-2}$ is the unitary sphere area in a $(d-1)$-dimensional spatial section of the spacetime, $S_{d-2}=2 \pi^{(d-1) / 2} / \Gamma((d-$ $1) / 2)$, where $\Gamma$ is the ordinary Gamma function and the factor $(d-2) G_{d} S_{d-2} /(d-3)$ reduces to $8 \pi G$ in four dimensions. The energy-momentum tensor $T_{\mu \nu}$ is given by

$$
T_{\mu \nu}=-p g_{\mu \nu}+(p+\rho) U_{\mu} U_{v}+\frac{1}{S_{d-2}}\left(F_{\mu}^{\gamma} F_{\gamma \mu}+\frac{1}{4} g_{\mu \nu} F_{\alpha \beta} F^{\alpha \beta}\right),
$$

where $\rho$ and $p$ are respectively the energy density and the pressure of the fluid in a $d$-dimensional spacetime, $F_{\mu \nu}$ is the electromagnetic field tensor, defined in terms of the potential $A^{\mu}$ through $F_{\mu \nu}=\nabla_{v} A_{\mu}-\nabla_{\mu} A_{v}$, with $\nabla_{v}$ is the covariant derivative. In equation (2.2), $J^{\mu}$ is the current density, with $J^{\mu}=\rho_{e} U^{\mu}$ where $\rho_{e}$ is the current density, and $U^{\mu}$ is the velocity of the fluid in a $d$-dimensional spacetime.

In order to describe a static fluid distribution with desired symmetry the line element is assumed to be of the following form:

$$
d s^{2}=H(r) d t^{2}-G(r) d r^{2}-r^{2} d \theta^{2}-r^{2} f(\theta)^{2} d \Sigma_{d-3}^{b},
$$

where $H$ and $G$ are function of $r$ only. The explicit form of the function $f(\theta)$ defines the geometry of the surface $S$ ( $r=$ constant, $t=$ constant): If $b=1$ then $f(\theta)=\sin \theta$ and $S$ is a $(d-1)$ dimensional sphere; if $b=-1$ then $f(\theta)=\sinh \theta$, the symmetry of $S$ is hyperbolic, and the topo$\operatorname{logy}$ is toroidal; and if $b=0$ then $f(\theta)=$ constant, the symmetry of $S$ is planar, and the topology can be a plane, a cylinder, or a torus. The quantity $d \Sigma_{d-3}^{b}$ stands for

$$
d \Sigma_{d-3}^{1}=d \theta_{2}^{2}+\ldots+\prod_{i=2}^{d-4} \sin ^{2} \theta_{i} d \theta_{d-3}^{2}, \quad d \Sigma_{d-3}^{-1}=d \theta_{2}^{2}+\ldots+\prod_{i=2}^{d-4} \sin ^{2} \theta_{i} d \theta_{d-3}^{2},
$$




$$
d \Sigma_{d-3}^{0}=d \theta_{2}^{2}+\ldots+d \theta_{d-3}^{2}
$$

respectively, for $b=1, b=-1$, and $b=0$.

Due to the symmetry of the spacetime, it does not allow the existence of magnetic fields. With this we have that the only nonzero components of $F^{\mu v}$ is $F^{t r}=-F^{r t}$, the other terms are identically zero. Hence, the nonvanishing component in equation (2.2), when $v=r$, gives

$$
q^{\prime}=S_{d-2} \frac{\rho_{e} r^{d-2} d r}{\sqrt{H(r)}}
$$

where $q$ is the electric charge inside $r, r$ being an arbitrary value of the radial coordinate. The prime ' represent the derivative with respect to $r$.

Considering the metric (2.4), the nonzero components of Einstein equations (2.1) are:

$$
\begin{gathered}
\frac{(d-2)(d-3) b}{2 r^{2}}+\frac{(d-2) H^{\prime}(r)}{2 r H^{2}(r)}-\frac{(d-2)(d-3)}{2 H(r) r^{2}}-\Lambda=G_{d} \frac{d-2}{d-3}\left(S_{d-2} \rho+\frac{q^{2}(r)}{2 r^{2(d-2)}}\right), \\
-\frac{(d-2)(d-3) b}{2 r^{2}}+\frac{(d-2)(d-3)}{2 H(r) r^{2}}+\frac{(d-2) G^{\prime}(r)}{2 r G(r) H(r)}+\Lambda=G_{d} \frac{d-2}{d-3}\left(S_{d-2} p-\frac{q^{2}(r)}{2 r^{2(d-2)}}\right) .
\end{gathered}
$$

Moreover, from Bianchi identities $\nabla_{\mu} T^{\mu v}=0$, it follows

$$
\frac{G^{\prime}}{G}=\frac{2 q q^{\prime}}{S_{d-2} r^{2(d-2)}(p+\rho)}-\frac{2 p^{\prime}}{p+\rho}
$$

To simplify the equations (2.7)-(2.9), we define

$$
H(r)^{-1}=b-\frac{2 G_{d} m(r)}{(d-3) r^{d-3}}+\frac{G_{d} q^{2}}{(d-3)^{2} r^{2(d-3)}}-\frac{2 \Lambda r^{2}}{(d-1)(d-2)},
$$

where $m(r)$ is the mass function, which, after equation (2.7) satisfies the relation:

$$
m^{\prime}=S_{d-2} \rho r^{d-2}+\frac{q q^{\prime}}{(d-3) r^{d-3}},
$$

which represents the conservation of the mass in a $d$-dimensional spacetime. Finally, substituting equation (2.8) into (2.9) it follows

$$
p^{\prime}=-(p+\rho)\left[\frac{S_{d-2} G_{d} p r}{(d-3)}-\frac{G_{d} q^{2}}{(d-3) r^{2 d-5}}+\frac{G_{d} m}{r^{d-2}}-\frac{2 \Lambda r}{(d-1)(d-2)}\right] H(r)+\frac{q q^{\prime}}{S_{d-2} r^{2(d-2)}},
$$

this is the TOV equation with electric charge and cosmological constant in a $d$-dimensional spacetime. This result is comparable: with [1] for $b=1, \Lambda=0$ and $d=4$; with [6, 7] for $b=1, q=0$, $\Lambda=0$ and $d=4$.

Therefore, the final system of equations to be solve are equations (2.6), (2.10), (2.11) and (2.12). This gives us a set of coupled differential equations which we need to solve simultaneously to get some result. The boundary conditions are the following: at the center of the star (where $r=0$ ) we put, as usual, $q(r)=0, m(r)=0, \rho(r)=\rho_{c}, p(r)=p_{c}$. The surface of the star is identified with the surface $r=R$, where the pressure $p(R)$ vanishes. As a matter of fact, we have more unknown variables than the number of equations in the system. To close the system one usually postulate and equation of state. In the present case, a further input is needed due to the presence of the electric charge (see bellow). For the numerical solution of these equations it is considered that $b=1$ (spherical symmetry) and $\Lambda=0$. The cases $b=0,-1$ do not have equilibrium solutions, and the effects of the cosmological constant will be investigated in future work. 


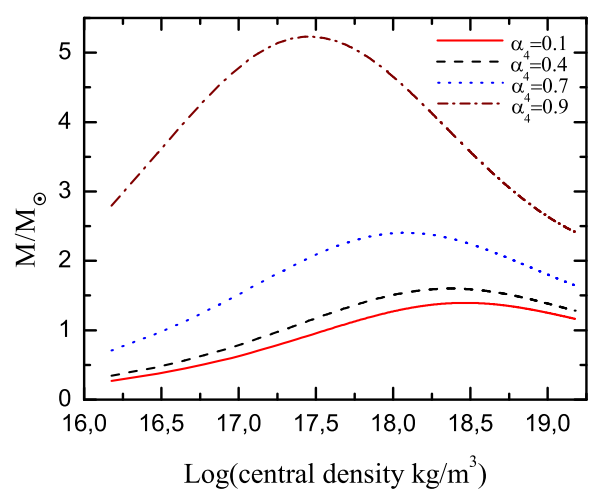

(a) The mass of the star as a function of the central density for different values of $\alpha_{4}$, and with the EOS $p_{4}=\omega_{4} \rho_{4}^{5 / 3}$.

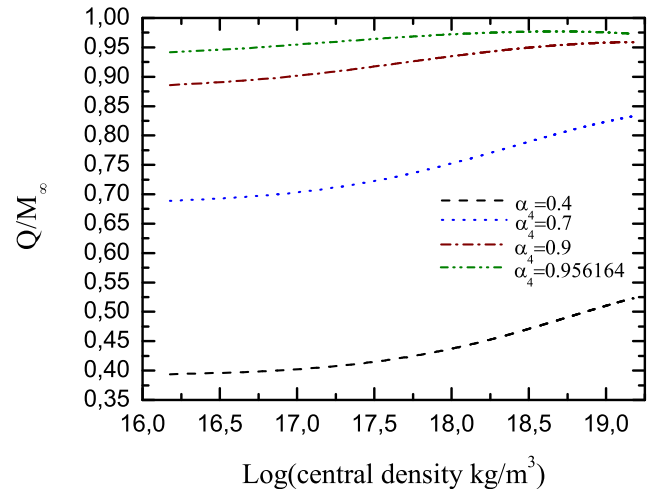

(b) Values of the ratio $Q / M_{\infty}$ against central density for different values of $\alpha_{4}$, and for the EOS $p_{4}=\omega_{4} \rho_{4}^{5 / 3}$.

\section{Numerical solution of the TOV equation}

In this section we study the effect of the electric charge in the structure of the charged cold stars by assuming that the charge density is proportional to mass-energy density (following [2]):

$$
\rho_{e}=\alpha_{d} \sqrt{G_{d}} \rho
$$

where $\alpha_{d}$ is a constant. In the CGS system of unit system, $\alpha_{d}$ is a non-dimensional constant in a $d$-dimensional spacetime.

\subsection{Charged star: polytropic equation of state}

The numerical calculations in this subsection is done in $d=4$, that is, in a four-dimensional spacetime. For comparison with other works, we use the following units: The mass density $\rho_{4}$ is given in $\left[\mathrm{MeV} / \mathrm{fm}^{3}\right]$, so that the Newton constant of gravitation $G$ is such that $\sqrt{G} \equiv \sqrt{G_{4}}$ bears the dimensions $\frac{1}{[\mathrm{~km}]}\left[\mathrm{fm}^{3} / \mathrm{MeV}\right]^{1 / 2}$. So, the charge density $\rho_{e 4}$ has the dimensions $\frac{1}{[\mathrm{~km}]}\left[\mathrm{MeV} / \mathrm{fm}^{3}\right]^{1 / 2}$.

The first polytropic EOS to be used here is of the form

$$
p_{4}=\omega_{4} \rho_{4}^{5 / 3}
$$

where the pressure has units of $\left[\mathrm{MeV} / \mathrm{fm}^{3}\right]$. Hence the units of the constant $\omega_{4}$ are $\left[\mathrm{fm}^{3} / \mathrm{MeV}\right]^{2 / 3}$ and the value considered for it is $\omega_{4}=1.41979 \times 10^{-3}$. The values for the central density in SI units are chosen in the interval $1.5 \times 10^{16} \mathrm{~kg} / \mathrm{m}^{3} \leq \rho_{c 4} \leq 1.5 \times 10^{19} \mathrm{~kg} / \mathrm{m}^{3}$.

With the EOS (3.2) and the values of the central density considered, we can analyze the system for different values of $\alpha_{4}$. In figure 1a we plot the mass as a function of the central density $\rho_{c 4}$ for different values of the charge fraction $\alpha_{4}$. The stars with large central density and low mass, are unstable because $\frac{d M}{d \rho_{c 4}}<0$ (see [8]). As we can see from figure 1a, the effect of the charge in the structure of the star for $\alpha_{4}=0.1$ is very small, and the curve is comparable with the star that does not have a net electric charge. When the value of $\alpha_{4}$ grows, until a critic value, the effect in the structure become important. To verify this let us consider an increment of about $300 \%$ in constant $\alpha_{4}$, from 0.1 to 0.4 . In this case, the maximum mass grows in approximately $15 \%$. However, an 
increment in $\alpha_{4}$ of just $30 \%$, from $\alpha_{4}=0.75$ to $\alpha_{4}=0.9$, causes a change in the maximum mass of almost $120 \%$. The changes in the structure in all cases is non-linear with the increase of the charge fraction as we can see in figure $1 \mathrm{a}$.

The values of the charge to mass ratio $\left(Q / M_{\infty}\right)$ in geometric units for different values of $\alpha_{4}$ in function of the mass central densities are shown in figure $1 \mathrm{~b}$. Such a ratio indicates how close the electric charge and the ADM mass (the mass of the star as seen by an observer at infinity). One then may look for a central energy density that leads to the extreme case, where $\rho_{e 4}=\rho_{4}$ and $Q=M_{\infty}$. Such a central density was not found in the present case.

\subsection{Charged star: polytropic equation of state depending on the charge density}

The studies made it in recently works of charged fluids in equilibrium indicate that the electric charge density play the role of pressure gradient $[5,9]$. In this sense, the electric charge density acts favorable to the pressure gradient, to counterbalance the gravitational attraction. Hence, it is reasonable to propose that the effective polytropic EOS of a charged fluid take the form $p_{4}=p_{4}\left(\rho_{4}, \rho_{e 4}\right)$. A simple form of such a relationship keeps the polytropic state equation in the form $p_{4}=\omega_{4} \rho_{4}^{5 / 3}-\beta \rho_{e 4}^{5 / 3}$, where $\beta$ is a non-negative parameter. Moreover, it is expected that an increase in the charge density implies a decrease in the pressure. According to this, and considering a relationship between $\rho_{e 4}$ and $\rho_{4}$ of the form (3.1), we may chose a polytropic EOS of the form

$$
p_{4}=\left(1-\alpha_{4}\right) \omega_{4} \rho_{4}^{5 / 3} \text {. }
$$

where $\alpha_{4}$ is an arbitrary constant.

With the EOS as in equation (3.3) and different values for the central density, the system can be analyzed for different values of $\alpha_{4}$. For comparison, the numerical values considered for $\alpha_{4}, \omega_{4}$ and $\rho_{c 4}$ are the same as in subsection 3.1.

The values of the mass of each star for different central densities are shown in figure 1a. Again the stars with large central density and low mass are unstable (see [8]). As in the case of the preceding section, the effects of the charge are noticeable just for sufficiently large values of $\alpha_{4}$. The main difference is that we find equilibrium solutions with larger charge factions. In fact, there are solutions for $\alpha_{4}$ as large as $\alpha_{4}=0.997888$.

The quantity $Q / M_{\infty}$, in geometric units, as a function of the central density, is shown in figure $1 \mathrm{~b}$ for different values of $\alpha_{4}$. We see that for the equation of state (3.3), there are equilibrium solutions for values of $Q / M_{\infty}$ closer to the case $\left(Q=M_{\infty}\right)$ than what was reported in the last subsection.

\subsection{Charged star in $d>4$ spacetimes, and with other symmetries $(b=0,-1)$}

We also studied equilibrium solutions for charged star in higher dimension, for the first EOS (3.2) of subsection 3.1. For a five-dimensional spacetime, the overall behavior of the curves are very similar to those obtained for $d=4$. The main different of these is the amount of the mass and radius size, that increase with the increment the number of extra dimensions. For $d=6$, we found only unstable solutions, and it was not possible to perform a detailed the analysis due to numerical convergence problems. A more careful study for $d=6$ and for high dimensions is being performed and will be reported elsewhere. 


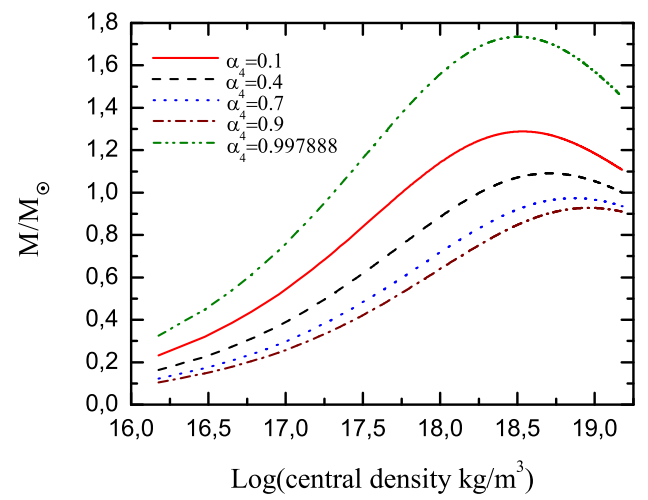

(a) Total mass of the star as a function of the central density for different values of $\alpha_{4}$ with the EOS $p_{4}=(1-$ $\left.\alpha_{4}\right) \omega_{4} \rho_{4}^{5 / 3}$.

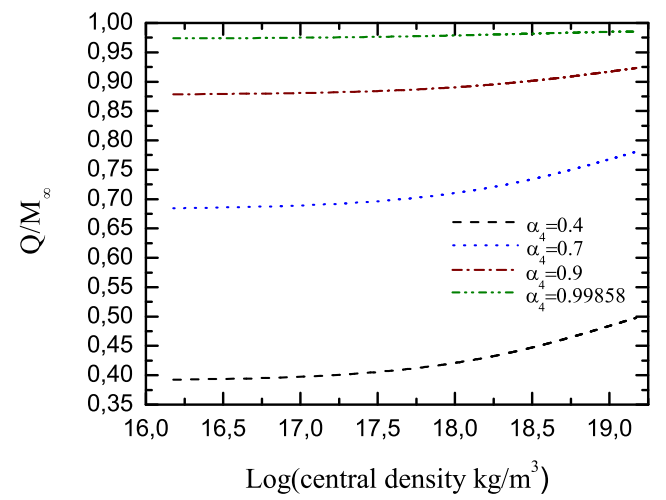

(b) $Q / M_{\infty}$ against the central density for different values of $\alpha_{4}$ with the polytropic EOS $p_{4}=\left(1-\alpha_{4}\right) \omega_{4} \rho_{4}^{5 / 3}$.

With the initial conditions used here to solve the TOV equation, it was not possible to find numerical solutions of equilibrium for the cases $b=0$ and $b=-1$. This means that, does not exist stars with planar and hyperbolic symmetry. In fact, it is possible to show that, for $b=0$ the right hand side of equation (2.12) assumes arbitrarily large positive values in the central region of the star, and for $b=-1$ the right hand side of (2.12) is also positive. Then, the pressure $p(r)$ increases monotonically (indefinitely) with the radial coordinate and cannot satisfy the condition $p(r)=0$ for a finite value of the coordinate $r$.

\section{References}

[1] J. Bekenstein, "Hydrostatic equilibrium and gravitational collapse of relativistic charged fluid balls", Phys. Rev. D 4, 2185 (1971).

[2] S. Ray, A.L. Espíndola, M. Malheiro, J.P.S. Lemos and V.T. Zanchin, "Electrically charged compact stars and formation of charged black holes", Phys. Rev. D 68, 084004 (2003).

[3] F. De Felice, L. Siming and Y. Yunqiang, "Relativistic charged spheres II. Regularity and stability", Class. Quantum. Grav. 16, 2669 (1999).

[4] T. Harko and M. Mak, "Anisotropic charged fluid spheres in D spacetime dimensions", J. Math. Phys. 41, 4752 (2000).

[5] J.P.S. Lemos and V.T. Zanchin, "Bonnor stars in d spacetime dimensions", Phys. Rev. D 77, 064003 (2008).

[6] J.R. Oppenheimer and G. Volkoff, "On massive neutron cores", Phys. Rev. 55, 374 (1939).

[7] R.C. Tolman, "Static solution of Einstein's field equation for spheres of fluid", Phys. Rev. 55, 364 (1939).

[8] S.L. Shapiro and S.A. Teukolsky, Black holes, white dwarfs and neutron: The physics of compact objects. (John Wiley \& Sons, New York, 1983).

[9] J.P.S. Lemos and V.T. Zanchin, "Electrically charged fluids with pressure in Newtonian gravitation and general relativity in d spacetime dimensions: Theorems and results for Weyl type system", Phys. Rev. D 80, 024010 (2009). 